Ef fi ci ent gener at i on of hi ghl y i mmonocompet ent dendritic cells fromperi pheral bl ood of pat i ent s wi th hepat i t i s C vi rus-rel at ed hepat ocel I ul ar car ci noma

\begin{tabular}{|c|c|}
\hline 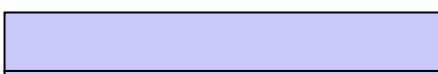 & 北原 征明 \\
\hline 著者別表示 & K t ahar a Masaaki \\
\hline $\begin{array}{l}\text { j our nal or } \\
\text { publ i cat i on } \mathrm{titl} \text { e }\end{array}$ & 博士論文本文Ful I \\
\hline 学位授与番号 & 13301乙第2062号 \\
\hline 学位名 & 博士 (医学) \\
\hline 学位授与年月日 & $2015-03-23$ \\
\hline URL & ht t p: //hdl . handl e. net /2297/43529 \\
\hline
\end{tabular}




\title{
Efficient generation of highly immunocompetent dendritic cells from peripheral blood of patients with hepatitis $C$ virus-related

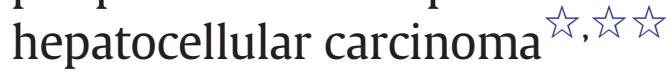

\author{
Masaaki Kitahara ${ }^{a}$, Eishiro Mizukoshi ${ }^{a}$, Yasunari Nakamoto ${ }^{b}$, Naofumi Mukaida ${ }^{c}$, \\ Kouji Matsushima ${ }^{\mathrm{d}}$, Shuichi Kaneko ${ }^{\mathrm{a}, *}$ \\ a Department of Gastroenterology, Graduate School of Medicine, Kanazawa University, Kanazawa, Japan \\ b Second Department of Internal Medicine, Faculty of Medical Sciences, University of Fukui, Fukui, Japan \\ c Division of Molecular Bioregulation, Cancer Research Institute, Kanazawa University, Kanazawa, Japan \\ d Department of Molecular Preventive Medicine, Graduate School of Medicine, University of Tokyo, Tokyo, Japan
}

\section{A R T I C L E I N F O}

\section{Article history:}

Received 13 April 2014

Received in revised form 19 May 2014

Accepted 21 May 2014

Available online 2 June 2014

\section{Keywords:}

Immunotherapy

$\mathrm{HCV}$

Cancer

Cytokine

\begin{abstract}
A B S T R A C T
Background \& aims: Immunotherapy using dendritic cells (DCs) is a promising cancer therapy. The success of this therapy depends on the function of induced DCs. However, there has been no consensus on optimal conditions for DC preparation in vitro for immunotherapy of hepatocellular carcinoma (HCC) patients. To address relevant issues, we evaluated the procedures to induce DCs that efficiently function in hepatitis C virus (HCV)-related HCC. Methods: We studied immunological data from 14 HCC patients. The DC preparation and the surface markers were assessed by flow cytometric analysis. Four different additional activation stimuli (Method I, medium alone; Method II, with OK-432; Method III, with IL-1 $\beta$ + IL-6 + TNF- $\alpha$; Method IV, with IL-1 $\beta$ + IL-6 + TNF$\alpha+$ PGE2) were tested and the functions of DCs were confirmed by examination of the ability of phagocytosis, cytokine production and allogeneic mixed lymphocyte reaction (MLR).

Results: The numbers of DCs induced and their cytokine production ability were not different between healthy controls and HCC patients. T-cell stimulatory activity of DCs in MLR was significantly lower in HCC patients than in healthy controls. The maturation of DCs with OK-432 boosted production of cytokines and chemokines, such as IL-2, IL-12p70, IFN- $\gamma$, TNF- $\alpha$, IL-13 and MIP1 $\alpha$, and restored T-cell stimulatory activity of DCs in MLR. Conclusions: The clinically approved compound OK-432 is a candidate for highly immunocompetent DC preparation and may be considered as a key drug for immunotherapy of HCV-related HCC patients.
\end{abstract}

(c) 2014 Published by Elsevier B.V.

\section{Introduction}

Hepatocellular carcinoma (HCC) is the seventh most common cancer worldwide and the fourth leading cause of cancer-related deaths in Japan [1]. Hepatitis C virus (HCV) infection is a common cause of

Abbreviations: DC, dendritic cell; HCV, hepatitis C virus; IFN, interferon; PBMC, peripheral blood mononuclear cell.

Grant support: Research grants from the Ministry of Education, Culture, Sports, Science and Technology of Japan.

弥弥 Author contributions: Masaaki Kitahara was responsible for analysis of the data and drafting of the article; Eishiro Mizukoshi was responsible for conception and design of the article; Yasunari Nakamoto, Naofumi Mukaida and Kouji Matsushima were responsible for critical revision of the article for important intellectual content; Shuichi Kaneko was responsible for final approval of the article.

* Corresponding author at: Department of Gastroenterology, Graduate School of Medicine, Kanazawa University, Kanazawa, Ishikawa 920-8641, Japan. Tel.: +8176265 2230; fax: +8176234 4250 .

E-mail address: skaneko@m-kanazawa.jp (S. Kaneko). chronic liver disease and leads to liver cirrhosis, contributing to the incidence of HCC [2]. Although some therapies for HCC exist, tumor recurrence rates are extremely high in these patients after curative treatments, including hepatic resection and radiofrequency ablation (RFA) [3]. In terms of the reason of this, HCV-related chronic hepatitis and cirrhosis are known to have carcinogenic potential for the development of HCC [4]. For the secondary chemoprevention of HCC patients with HCVrelated cirrhosis, recent studies showed the efficacy of long-term and low-dose interferon- $\alpha$ therapy [5]. Among many strategies for HCC recurrence, immunotherapy is considered to be an attractive strategy to eradicate tumor cells completely [6].

Until now, different immunotherapeutic approaches have been tested for patients with HCC [7]. However, tumors have evolved numerous immune escape mechanisms, including the generation of cells with immune suppressor functions, such as Tregs and myeloid-derived suppressor cells [8]. Dendritic cells (DCs) are the most potent professional antigen-presenting cells with the unique ability to initiate and maintain adaptive immunity, and are considered 
to overcome the immune-suppressive environment produced by tumors $[9,10]$. DCs are also known to enhance antitumor immunity by activating the functions of endocytosis, trafficking, maturation and cytokine production. Numerous studies have shown that DCs from peripheral blood of HCC patients are reduced in number, have an immature phenotype and an impaired function [11]. Moreover, to date, there is no consensus on optimal conditions for DC preparation in vitro for immunotherapy of HCC patients.

Recently, we have developed the combined immunotherapy of transcatheter hepatic arterial embolization (TAE) with infusion of immature and mature monocyte-derived DCs (MoDCs) for HCC [12, 13]. In this study, we evaluated the procedures to induce MoDCs that efficiently function in the immune-mediated treatments for HCC.

\section{Materials and methods}

\subsection{Patients and healthy controls}

Fourteen patients (four women and ten men) attending Kanazawa University Hospital (Ishikawa, Japan) between September 2007 and December 2008 were enrolled in this study. All patients were serologically positive for HCV. HCC was radiologically diagnosed by computed tomography (CT), magnetic resonance imaging (MRI) and CT angiography. Blood samples were taken from 14 patients with HCC and 14 healthy controls having no hepatitis history and serologically negative for both hepatitis B and C. The clinical profiles of the patients and controls analyzed in the present study are shown in Table 1. All patients gave written informed consent to participate in the study in accordance with the Helsinki Declaration and this study was approved by the regional ethics committee (Medical Ethics Committee of Kanazawa University, No. 829).

\subsection{Preparation of immature $D C S$}

Immature DCs were separated from peripheral blood mononuclear cells (PBMCs) of patients and healthy controls. PBMCs were isolated by centrifugation using Lymphoprep ${ }^{\mathrm{TM}}$ Tubes (Nycomed, Roskilde, Denmark). The cells were resuspended in serum-free medium (GMP CellGro DC Medium; CellGro, Manassas, VA) and allowed to adhere to 6-well tissue culture dishes (Costar, Cambridge, MA) at $1.4 \times 10^{7}$ cells in $2 \mathrm{~mL}$ per well. After $2 \mathrm{~h}$ at $37^{\circ} \mathrm{C}$, non-adherent cells were removed and adherent cells were cultured in the medium with $50 \mathrm{ng} / \mathrm{mL}$ recombinant human IL-4 (GMP grade; CellGro ) and $100 \mathrm{ng} / \mathrm{mL}$ recombinant human GM-CSF (GMP grade; CellGro ) for 5 days to generate immature DCs.

Table 1

Characteristics of healthy controls and patients.

\begin{tabular}{llll}
\hline & Controls & HCC patients & $P$ \\
\hline No. of patients & 14 & 14 & \\
Age (years) & $42.6 \pm 14.9$ & $68.8 \pm 7.6$ & $<.05$ \\
Gender $(\mathrm{M} / \mathrm{F})$ & $7 / 7$ & $10 / 4$ & n.s. \\
WBC $\left(\mathrm{X} 10^{2} / \mathrm{LL}\right)$ & ND & $43.5 \pm 15.4$ & n.s. \\
PLT $\left(\mathrm{X} 10^{4} / \mu \mathrm{L}\right)$ & ND & $13.1 \pm 6.0$ & n.s. \\
PT $(\%)$ & ND & $85.2 \pm 13.3$ & n.s. \\
ALT $(\mathrm{IU} / \mathrm{L})$ & ND & $59.7 \pm 46.8$ & n.s. \\
Alb $(\mathrm{g} / \mathrm{dL})$ & ND & $3.3 \pm 0.6$ & n.s. \\
T-Bil $(\mathrm{mg} / \mathrm{dL})$ & ND & $0.8 \pm 0.4$ & n.s. \\
Histology of non-tumor liver & & & \\
Chronic hepatitis & ND & 8 & n.s. \\
Cirrhosis $($ Child-Pugh A/B/C) & ND & $6(5 / 1 / 0)$ & n.s. \\
TNM stage (I/II/III/IIIB/IIIC/IV) & ND & $0 / 11 / 1 / 2 / 0 / 0$ & \\
\hline
\end{tabular}

Data are expressed as the mean \pm SD.

ND: not determined, n.s.: not significant.
Induction of MoDC

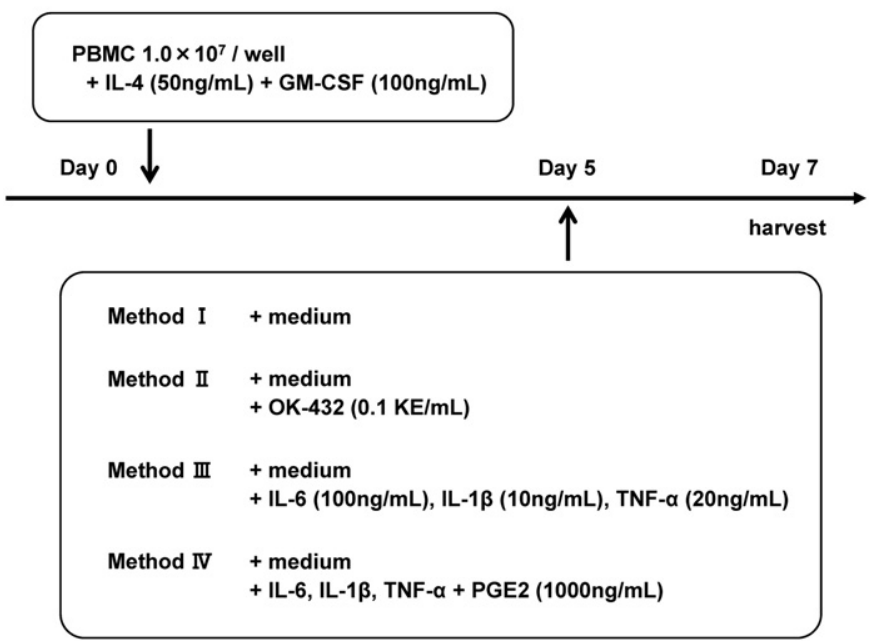

Fig. 1. Protocols for preparation of DCs. DCs, which were derived from PBMCs in the presence of IL-4 and GM-CSF for 5 days, were cultured for 2 additional days with the serum-free medium only (Method I), OK-432 (Method II), and cytokine cocktails consisting of IL-6, IL-1 $\beta$ and TNF- $\alpha$ without (Method III) or with (Method IV) PGE2. On day 7 , these cells were harvested and evaluated.

\subsection{Activation of immature DCS}

Several activation stimuli were tested (Fig. 1). After 5 days of culture, the immature DCs induced by the above method were cultured for 2 additional days in the serum-free medium (Method I) or stimulated with $0.1 \mathrm{KE} / \mathrm{mL}$ OK-432 (Chugai Pharmaceuticals, Tokyo, Japan) (Method II), 10 ng/mL IL-1ß (GMP grade; CellGro ), 100 ng/mL IL-6 (GMP grade; CellGro ) and $20 \mathrm{ng} / \mathrm{mL}$ TNF- $\alpha$ (GMP grade; CellGro ) (Method III), and IL-1 $\beta$, IL-6, TNF- $\alpha$ and PGE2 (Kaken Pharmaceuticals, Tokyo, Japan) (Method IV). On day 7, the cells were harvested.

\subsection{Antibodies}

The following anti-human monoclonal antibodies (mAbs) were used for flow cytometry: anti-lin1 (lineage cocktail 1; CD3, CD14, CD16, CD19, CD20 and CD56)-FITC, anti-HLA-DR-PerCP and -FITC (L243), anti-CD11C-APC (S-HCL-3), anti-CD123-PE (9F5), anti-CCR7PE (3D12), anti-CD14-APC (M $\varphi$ P9) (BD Biosciences Pharmingen, San Jose, CA), anti-CD80-PE (MAB104), anti-CD83-PE (HB15a) and anti-CD86-PE (HA5.2B7) (Beckman Coulter, Fullerton, CA).

\subsection{Flow cytometric analysis}

Surface markers on DCs were evaluated using flow cytometric analysis. Cells were analyzed on a FACSCalibur ${ }^{\mathrm{TM}}$ for four-color flow cytometry. Data analysis was performed using CELLQuest ${ }^{\mathrm{TM}}$ software (Becton Dickinson, San Jose, CA).

\subsection{Endocytosis assay}

The endocytic capacity of DCs was assessed by measurement of FITC dextran (Sigma-Aldrich, St. Louis, MO) uptake. DCs were incubated for $30 \mathrm{~min}$ at $37{ }^{\circ} \mathrm{C}$ in the presence of $1 \mathrm{mg} / \mathrm{mL}$ FITC dextran, washed three times and analyzed using a FACSCalibur ${ }^{\mathrm{TM}}$ cytometer.

\subsection{Cytokine production assay}

The concentrations of cytokines and chemokines in the supernatants of culture medium were measured using enzyme-linked immunosorbent assay (ELISA) kit for IL-12p40 and IFN $\gamma$ (Biosource International, 
Camarillo, CA) and the Bioplex assay (Bio-Rad, Hercules, CA) according to the manufacturers' instructions.

\subsection{Allogeneic mixed lymphocyte reaction (allo-MLR)}

To evaluate the immune-stimulatory function of DCs after different stimulations, allogeneic mixed lymphocyte reaction (allo-MLR) was performed. $1 \times 10^{4}$ irradiated DCs $(25 \mathrm{~Gy})$ and $1 \times 10^{5}$ allogeneic PBMCs from a healthy donor were suspended in $200 \mu \mathrm{L}$ of serum-free medium (GMP CellGro DC Medium) and co-cultured in 96-well round-bottomed microplates. After 2 days at $37{ }^{\circ} \mathrm{C}, 1 \mu \mathrm{Ci}$ of $\left[{ }^{3} \mathrm{H}\right]$-thymidine (Amersham Pharmacia Biotech, Piscataway, New Jersey) was added to each well. The amount of incorporated $\left[{ }^{3} \mathrm{H}\right]$-thymidine was counted using a liquid scintillation counter (Beckman Coulter, Palo Alto, California). Results are expressed as the stimulation index (counts per minute in the presence of DCs divided by counts per minute in the absence of DCs).

A

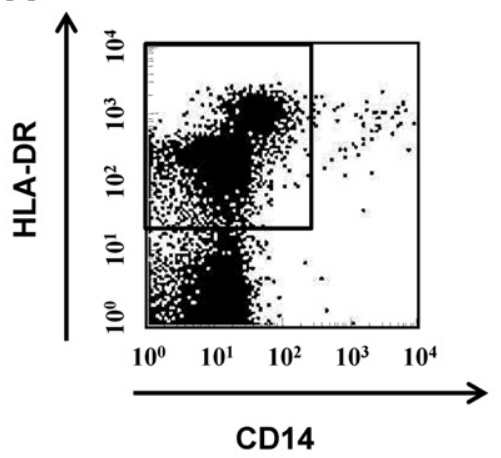

B

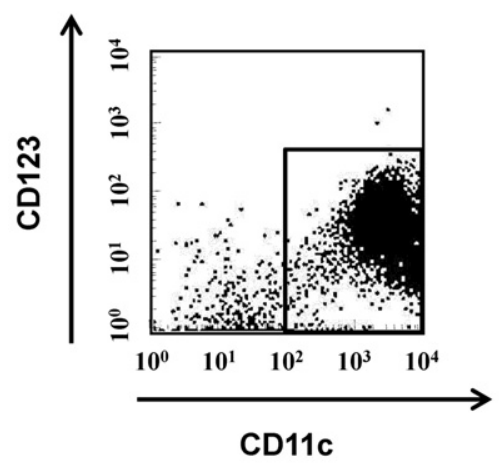

C

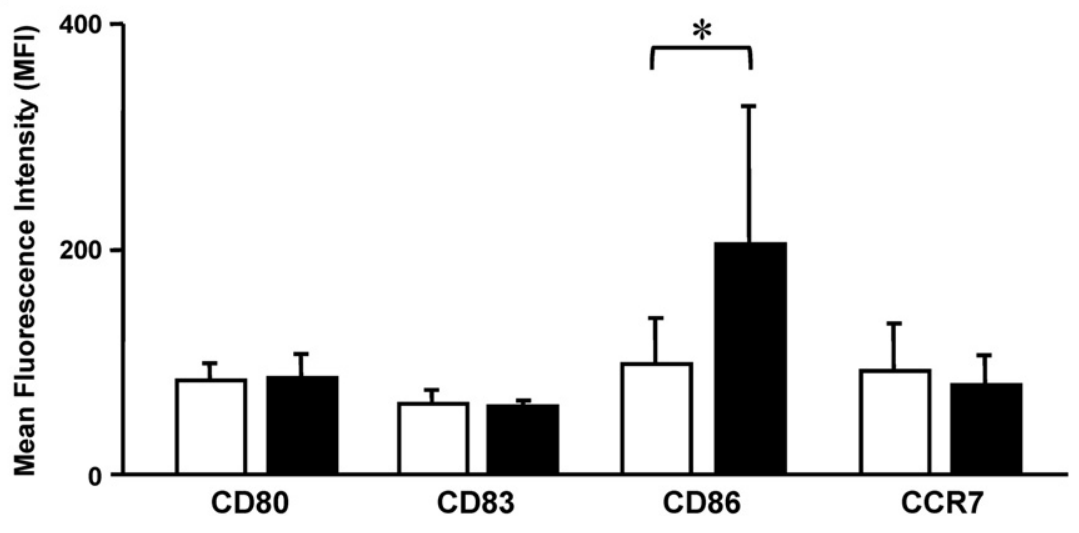
considered statistically significant.

\section{Results}
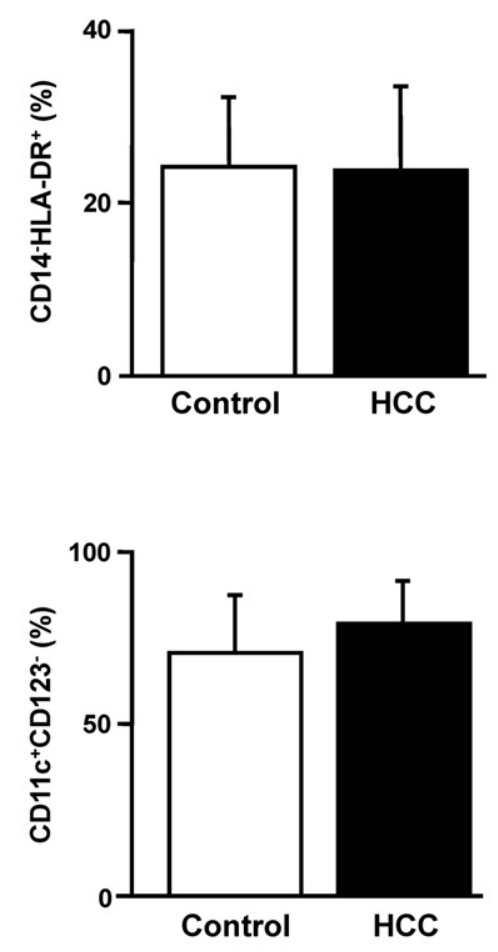

Results are indicated as means \pm SD. The statistical significance of differences between groups was determined by applying the MannWhitney $U$ test and unpaired $t$ test. Any $P$ values less than 0.05 were

\subsection{Yield and phenotype of DCs in patients and healthy controls}

Adherent cells separated from PBMCs were cultured in the presence of IL-4 and GM-CSF, and harvested on day 7 (Method I). These harvested cells, which showed high levels of MHC class II (HLA-DR) and the absence of marker for mature monocytes (CD14), were consistent with the cell surface markers of DCs. The yield of DCs was variable, ranging from $23 \%$ to $28 \%$ of the initial PBMC population, and indicated no 
significant difference between healthy controls and HCC patients (Fig. 2A). We next analyzed the surface markers, which classified DCs as myeloid or plasmacytoid DCs, on these lineage marker (lin1)-negative and HLA-DR-positive (lin $1^{-}$HLA-DR ${ }^{+}$) DCs. In both patients and controls, the majority of DCs expressed CD11c and the percentages of the DCs classified under the myeloid subset $\left(\mathrm{CD} 11 \mathrm{c}^{+} \mathrm{CD} 123^{-}\right)$were not different among the two groups (Fig. 2B).

Subsequently, we analyzed the expression of co-stimulatory molecules, including B7-1 (CD80), B7-2 (CD86), an activation marker (CD83) and a chemokine receptor (CCR7) by recording geometric mean fluorescence intensities (MFIs) (Fig. 2C). Notably, the expression level of CD86 was significantly increased in HCC patients (204.5 \pm $120.5, P<.05)$ compared with that of healthy controls $(97.7 \pm 42.3)$. The expression levels of CD80, CD83 and CCR7 were similar in the two groups.

\subsection{Functions of DCs in patients and healthy controls}

The endocytic and phagocytic capacities of DCs were assessed by measurement of FITC dextran uptake using flow cytometry. A representative result is shown in Fig. 3A. MFIs of DCs induced from PBMCs of HCC patients indicate more FITC dextran uptake than those from healthy controls $(223.3 \pm 82.9$ vs. $113.1 \pm 35.4, P<.05)$.

Next, we analyzed their ability to produce inflammatory cytokines that influence T-cell function. The spontaneous IL-12p40 production of DCs was measured using an enzyme-linked immunosorbent assay (ELISA) (Fig. 3B). The concentrations of IL-12p40 in the supernatant did not differ between HCC patients and healthy controls (11.4 \pm 3.2 vs. $14.2 \pm 7.5, P=.29$ ).

In contrast, regarding T-cell stimulatory activity of DCs in allogeneic MLR, which was expressed as stimulation index value of $\left[{ }^{3} \mathrm{H}\right]$-thymidine incorporation at the T-cell/DC ratio of $10 / 1$, the index values were significantly lower in HCC patients than in healthy controls $(1.9 \pm 1.1$ vs. $3.5 \pm 0.5, P<.05$ ) (Fig. 3 C).

\subsection{Yield and phenotype of DCs with different stimulations}

Considering effective antitumor immunity of DC-based immunotherapy, maturational status and T-cell stimulatory potential of DCs are important. Therefore, we next examined the effects of different maturation stimuli on the phenotypes and the functions of DCs induced from PBMCs in HCC patients. As shown in Fig. 1, PBMCs of HCC patients were differentiated into immature DCs in the presence of IL-4 and GMCSF on day 5 and then harvested on day 7 after culturing for two additional days in several activation stimuli.

First, the expressions of HLA-DR, various costimulatory molecules and chemokine receptor, which were the indicators of DC maturation, were assessed (Fig. 4). By culturing with the additional stimuli (Methods II, III and IV), the percentage of CD14 ${ }^{-} \mathrm{HLA}^{-D R^{+}}$DCs was significantly increased compared with that in the medium alone (Method I) (Fig. 4A). However, the percentage of $\mathrm{CD} 11 \mathrm{c}^{+} \mathrm{CD} 123^{-}$DCs was not different in all groups.

In the next step, we assessed the geometric mean fluorescence intensities of CD80, CD83, CD86 and CCR7 (Fig. 4B). The expressions of CD80 and CD86 of DCs with the stimulation (Methods II, III and IV) increased significantly compared with those with medium alone (Method I). Furthermore, the DCs stimulated with OK-432 (Method II) or cytokine cocktail with PGE2 (Method IV) showed a significant increase of CD80 and CD86 in comparison with those stimulated with cytokine cocktail without PGE2 (Method III). The expression level of CD83 was increased by OK-432 (Method II) and cytokine cocktail with PGE2
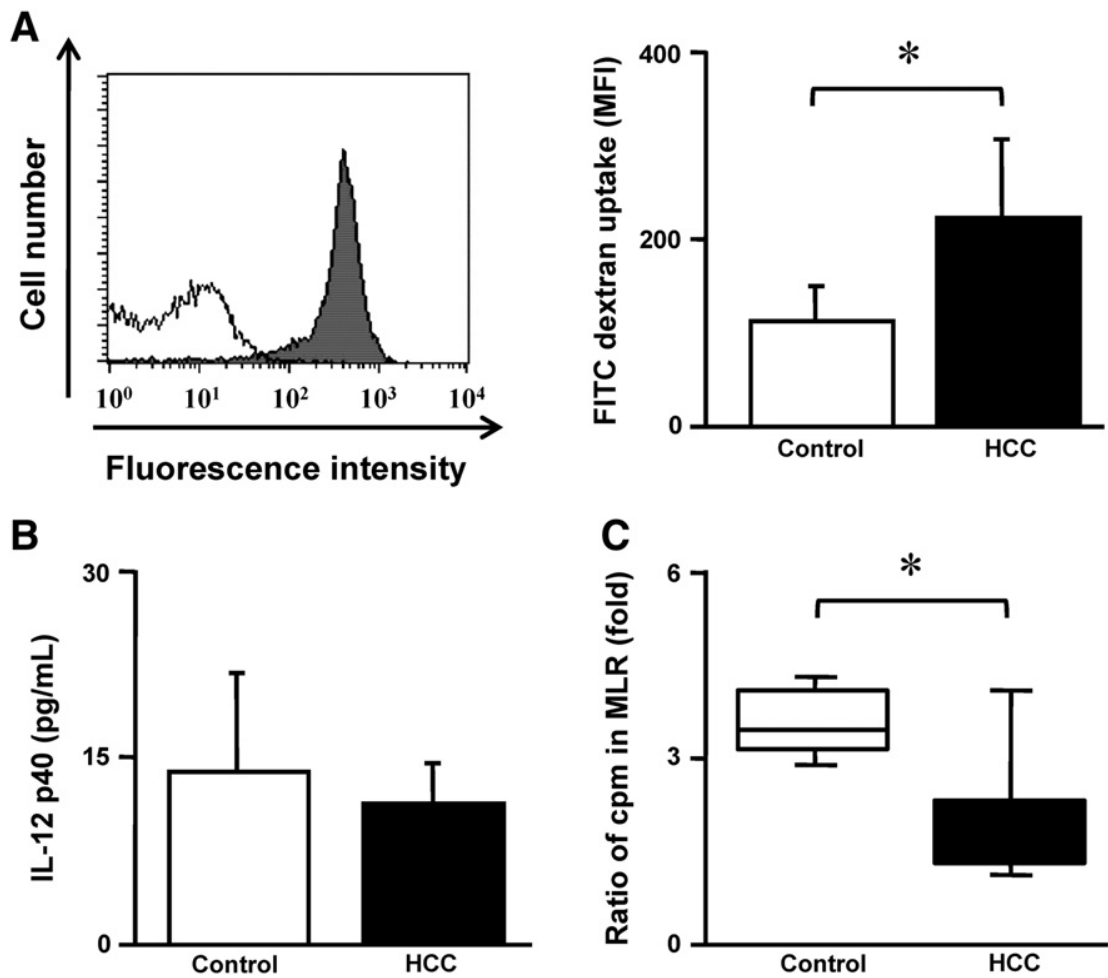

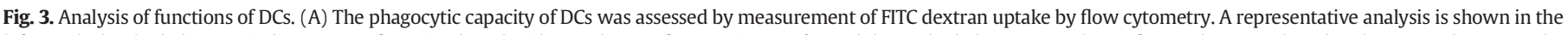

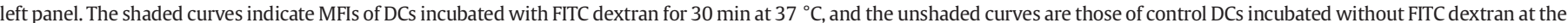

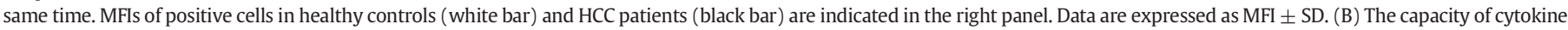

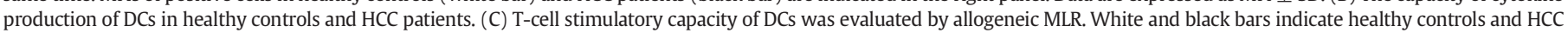
patients, respectively. ${ }^{*} \mathrm{P}<.05$. 
A
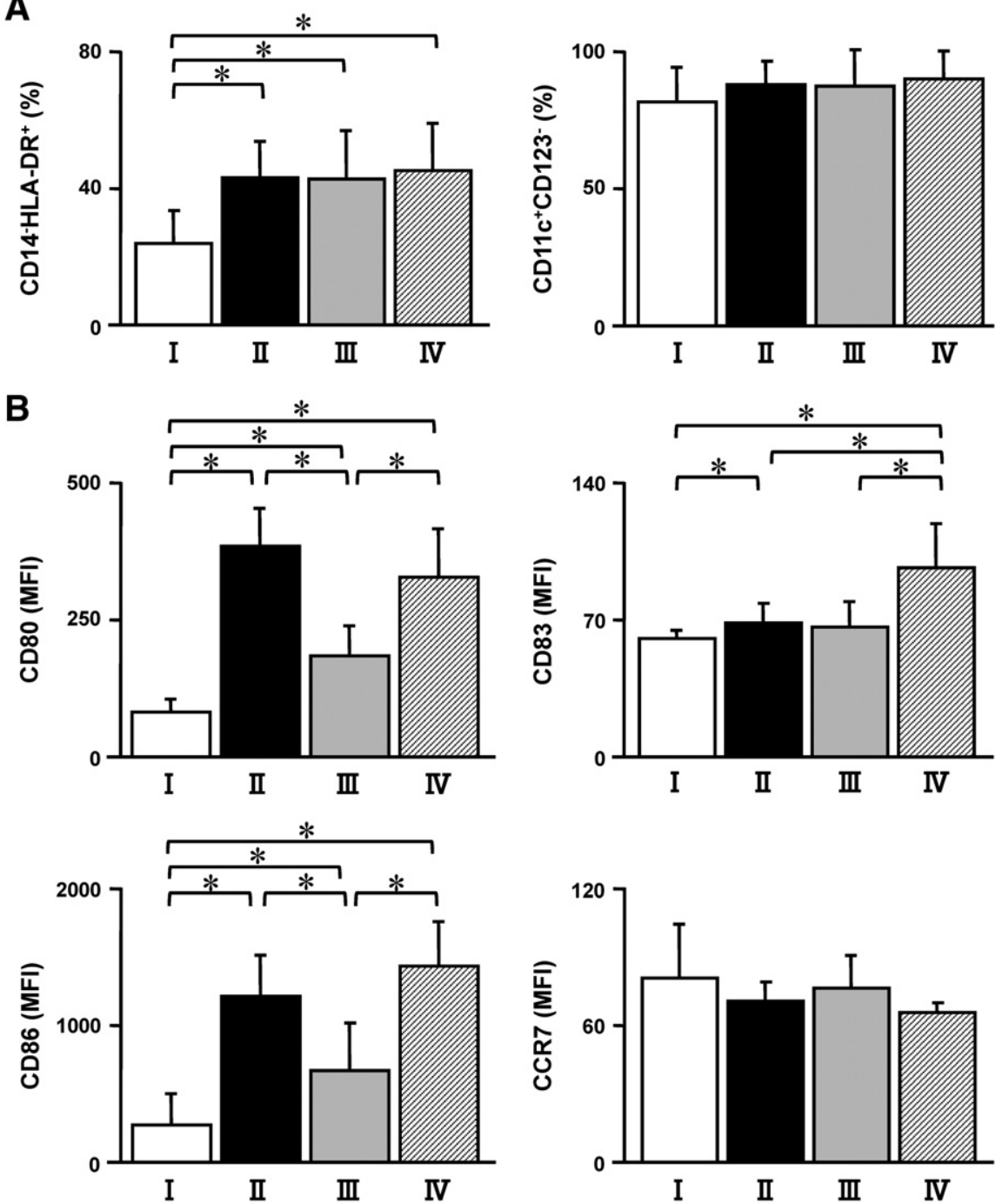

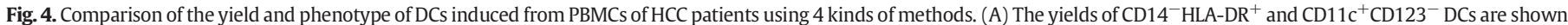

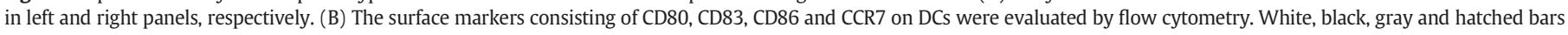
indicate the methods of DC maturation with methods I, II, III and IV, respectively. The methods of DC maturation are shown in Fig. $1 .{ }^{*} P<.05$.

(Method IV) stimulation. The expression of CCR7 was not different among the groups.

\subsection{Function of DCs with different stimulations}

Initially, the change of phagocytic capacity of DCs induced from PBMCs in HCC patients was assessed by the same protocol (shown above) (Fig. 5A). The uptake of FITC dextran of DCs stimulated with OK-432, cytokine cocktail with and without PGE2 (Methods II, III and IV) was decreased significantly compared with that of DCs cultured in medium alone (Method I) $(P<.05)$.

Next, we also examined cytokine production, such as IL-12p40 and IFN- $\gamma$, of DCs by ELISA (Fig. 5B). The DCs stimulated with OK-432 (Method II) produced much more IL-12p40 and IFN- $\gamma$ than the DCs stimulated with cytokine cocktail with and without PGE2 (Methods IV and III, respectively) or medium alone (Method I) $(P<.05)$. In the analysis of IFN- $\gamma$ production, the DCs stimulated with OK-432 (Method II) produced the largest amount of IFN- $\gamma$ among the groups $(P<.05)$.

\subsection{Allo-stimulatory capacity of DCs with different stimulations}

To assess whether the enhanced expression of costimulatory molecules reflects the antigen presentation capacity, we studied the function using an allo-MLR (Fig. 5C). Stimulation with OK-432 (Method II) or cytokine cocktail with PGE2 (Method IV) was more efficient in inducing T-cell proliferation than that with cytokine cocktail without PGE2 (Method III) or medium alone (Method I). Moreover, the index value of DCs stimulated with OK-432 was significantly higher than that of DCs stimulated with cytokine cocktail with PGE2 (9.9 \pm 3.9 vs. $4.7 \pm$ $1.7, P<.05)$.

To evaluate the mechanism of strong allo-stimulatory capacity of DCs induced by OK-432 in HCC patients, the cytokine levels in allogeneic MLR supernatant were examined using Bioplex assay (Fig. 6). The levels of cytokines in the medium containing DCs with OK-432 stimulation (Method II), such as IL-2, IL-12p70, IFN- $\gamma$, TNF- $\alpha$, IL-13 and MIP1 $\alpha$, were significantly higher than those in the medium containing DCs with medium alone (Method I) $(P<.05)$ (Fig. 6A). In addition to an increase of these cytokines and chemokines, other cytokines including IL-4, IL-10 and IL-17 were also significantly increased in the medium in which DCs with OK-432 stimulation and PBMCs were co-cultured (Fig. 6B).

\section{Discussion}

Immunotherapy is a promising therapy for HCC patients and a number of the therapies have been evaluated [14]. Among the numerous 

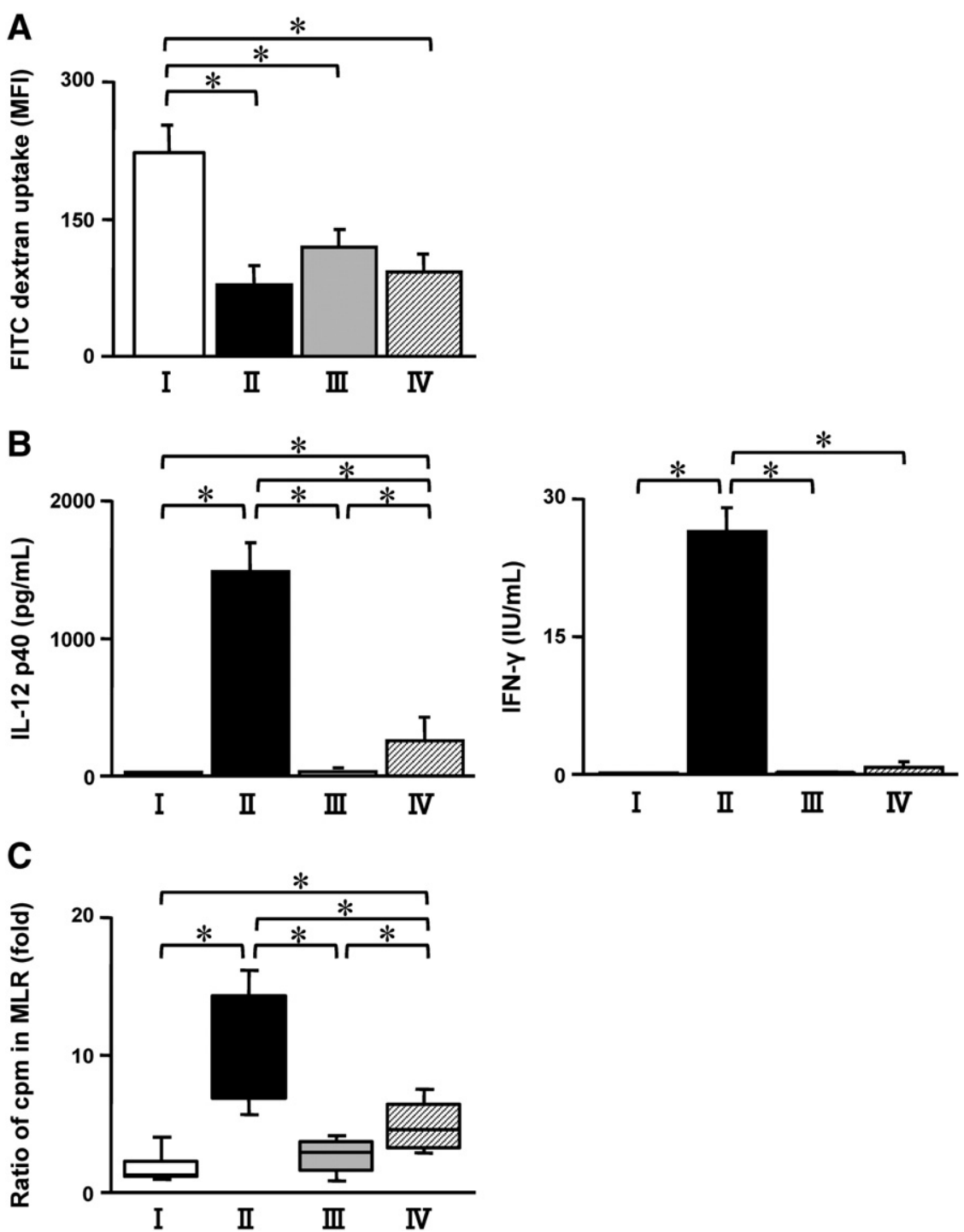

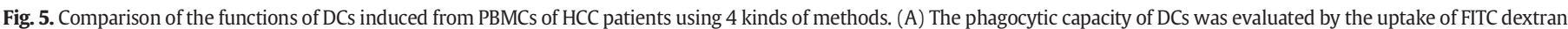

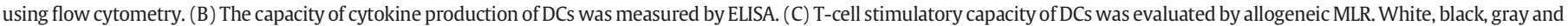
hatched bars indicate the methods of DC maturation with methods I, II, III and IV, respectively. The methods of DC maturation are shown in Fig. 1 . *P $<.05$.

immune cells, DCs are the most potent type of antigen-presenting cells in the human body. However, impaired function of DCs has been implicated in the escape of the tumor from immune control in cancer patients [15]. In chronic HCV-infected patients, recent studies have shown that the function of MoDCs is not necessarily impaired [16], while several groups have reported a maturation defect and impaired function in HCC patients [17].

In the present study, the number and cell surface maturation markers of harvested DCs derived from PBMCs of HCC patients are not different from those of healthy controls. In particular, the results that the percentage of $\mathrm{CD} 11 \mathrm{c}^{+} \mathrm{CD} 123^{-}$myeloid DCs was not different suggested that the culture method using IL-4 and GM-CSF is suitable for the induction of effective DCs in HCC immunotherapy. Because myeloid DC is a main player that produces cytokines such as IL-12, induces T-helper type 1 (Th1) response and antigen-specific cytotoxic Tcell immunity [18].

In the analysis of maturation markers, the DCs in HCC patients showed similar expression levels of CD80, CD83, CCR7 and even a high expression level of CD86 compared with the DCs in healthy donors. However, the result of FITC dextran uptake indicated that the DCs in
HCC patients showed high ability, suggesting that their functional phenotype is still immature. Consistent with these results, stimulatory capacity of these DCs in MLR was lower than that of healthy controls, suggesting that additional treatment is required for optimal DC preparation.

Unfortunately, there is still no consensus on the optimal procedure for preparation of DCs using the drugs with good manufacturing practice (GMP) grade. For the clinical application of DC-based immunotherapy, it is desirable to use maturation agents with GMP grade for the safety of patients. Therefore, in this study, we evaluated the procedures using GMP-grade compounds. OK-432, a penicillin-inactivated and lyophilized preparation of Streptococcus pyrogenes, was reported to be immunomodulatory and have potential therapeutic properties for cancer immunotherapy $[19,20]$. DCs stimulated with OK-432 have been suggested to acquire a mature phenotype, produce a significant amount of T-helper type 1 (Th1) cytokines such as IL-12 and IFN $\gamma$ and enhance cytotoxic T-lymphocyte activity [21]. Otherwise, many cytokines, often containing pro-inflammatory mediators, or their combinations have been tested for DC maturation. Since 1997, cytokine cocktails containing TNF- $\alpha$, IL-1 $\beta$, IL-6 and PGE2 have been shown to induce DC maturation 
A
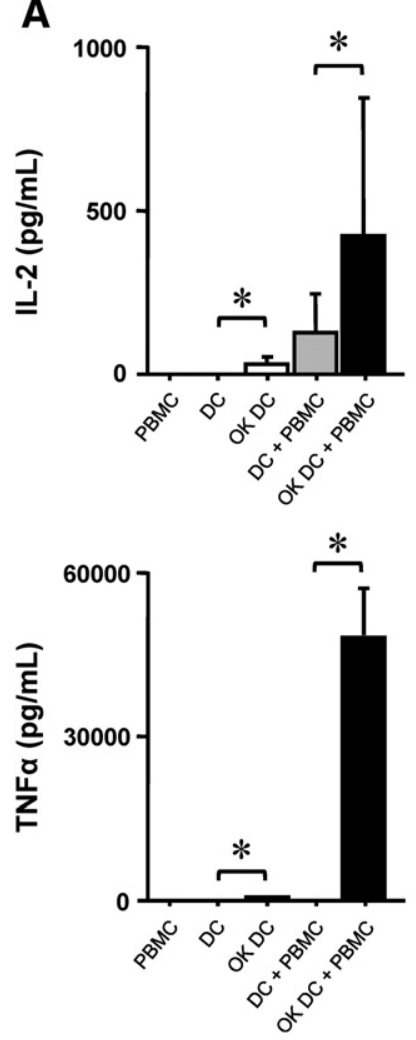

B

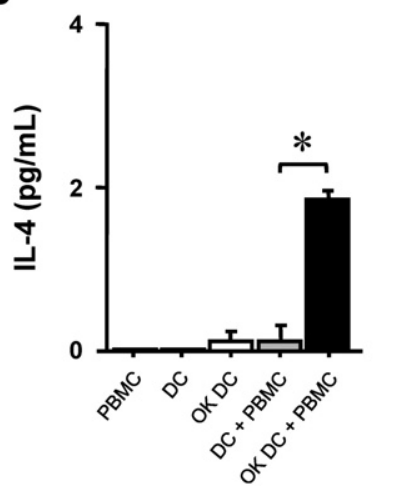

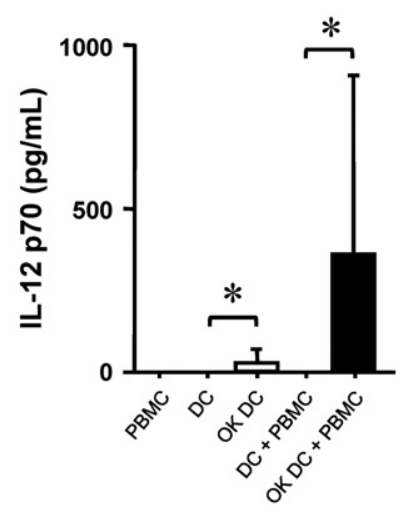
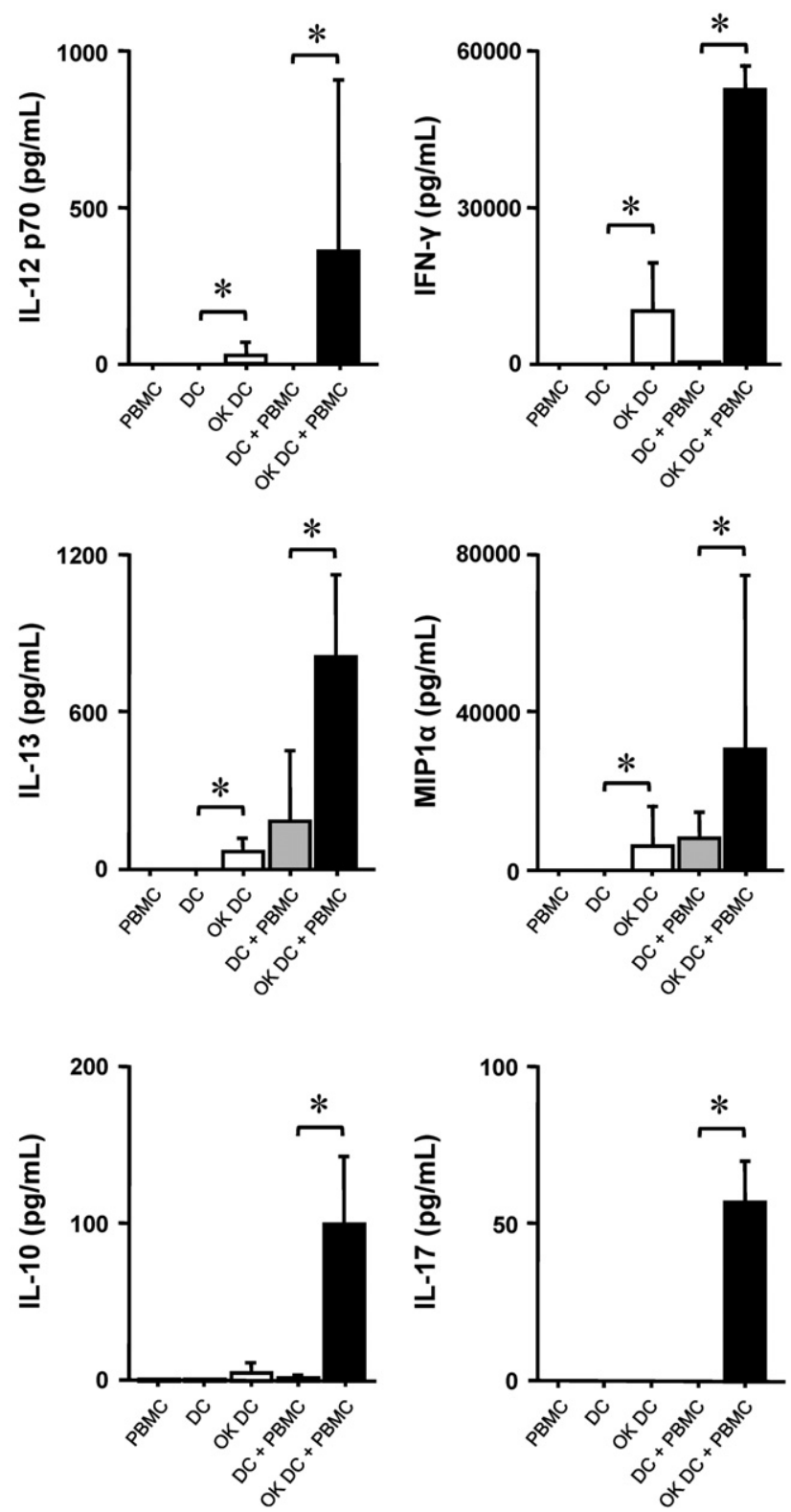

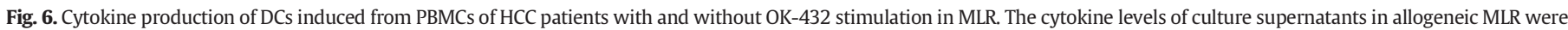

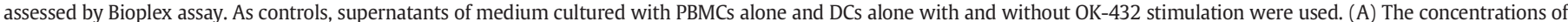

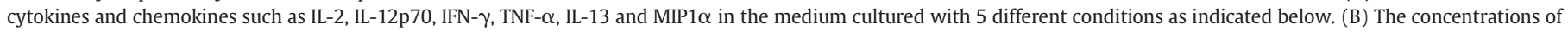

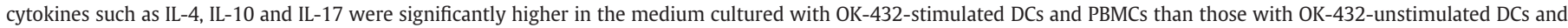

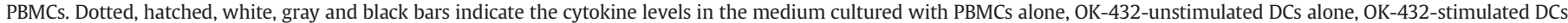
alone, OK-432-unstimulated DCs with PBMCs and OK-432-stimulated DCs with PBMCs, respectively. ${ }^{*} P<.05$.

[22]. In addition, adding PGE2 to the maturation cocktail was reported to enhance further CCR7 expression, migration capacity and T-cell stimulatory activity of DCs even in patients with advanced cancer [23]. In this study, on the basis of these results, we analyzed DCs derived from PBMCs of HCV-related HCC patients by OK-432 or cytokine cocktails.

Both methods, using OK-432 or cytokine cocktails, had success in enhancing the expression levels of CD80, CD83 and CD86. The phagocytic capacity of the DCs induced by both methods was lower than that of the DCs induced by a standard method. On the other hand, the production of cytokines such as IL-12p40 and IFN- $\gamma$ and allostimulatory capacity were excellent in DCs with OK-432 stimulation. These results suggest that both methods are useful for maturation, but OK-432 stimulation is the best method of DC preparation for immunotherapy in HCV-related HCC patients. Moreover, the types of cytokines and chemokines detected in allogeneic MLR were very similar to those that we previously reported in serum of patients who received immunotherapy with OK-432-stimulated DCs [13]. Taken together with these results, the DCs stimulated with OK-432 may have immunological potential in not only local but also systemic responses through cytokine production.

In conclusion, the results of the present study suggest that the clinically approved compound OK-432 is a candidate for highly immunocompetent DC preparation in HCV-related HCC patients and should provide us with a novel insight for immunotherapy of HCC.

\section{Acknowledgments}

The authors thank Mariko Katsuda, Kazumi Fushimi and Maki Kawamura for technical assistance. We also thank the patients for participating in this study. This work was supported in part by research 
grants from the Ministry of Education, Culture, Sports, Science and Technology of Japan.

\section{References}

[1] Kudo M. Real practice of hepatocellular carcinoma in Japan: conclusions of the Japan Society of Hepatology 2009 Kobe Congress. Oncology 2010;78(Suppl. 1):180-8.

[2] Lok AS, Seeff LB, Morgan TR, di Bisceglie AM, Sterling RK, Curto TM, et al. Incidence of hepatocellular carcinoma and associated risk factors in hepatitis C-related advanced liver disease. Gastroenterology 2009;136:138-48.

[3] Tiong L, Maddern GJ. Systematic review and meta-analysis of survival and disease recurrence after radiofrequency ablation for hepatocellular carcinoma. Br J Surg 2011;98:1210-24.

[4] El-Serag HB, Rudolph KL. Hepatocellular carcinoma: epidemiology and molecular carcinogenesis. Gastroenterology 2007;132:2557-76.

[5] Sakaguchi Y, Kudo M, Fukunaga T, Minami Y, Chung H, Kawasaki T. Low-dose, longterm, intermittent interferon-alpha-2b therapy after radical treatment by radiofrequency ablation delays clinical recurrence in patients with hepatitis $C$ virus-related hepatocellular carcinoma. Intervirology 2005;48:64-70.

[6] Butterfield LH. Immunotherapeutic strategies for hepatocellular carcinoma. Gastroenterology 2004;127:S232-41.

[7] Greten TF, Manns MP, Korangy F. Immunotherapy of HCC. Rev Recent Clin Trials 2008;3:31-9.

[8] Serafini P, Borrello I, Bronte V. Myeloid suppressor cells in cancer: recruitment, phenotype, properties, and mechanisms of immune suppression. Semin Cancer Biol 2006;16:53-65.

[9] Banchereau J, Steinman RM. Dendritic cells and the control of immunity. Nature 1998;392:245-52.

[10] Palucka K, Banchereau J. Dendritic cells: a link between innate and adaptive immunity. J Clin Immunol 1999;19:12-25.

[11] Kakumu S, Ito S, Ishikawa T, Mita Y, Tagaya T, Fukuzawa Y, et al. Decreased function of peripheral blood dendritic cells in patients with hepatocellular carcinoma with hepatitis B and C virus infection. J Gastroenterol Hepatol 2000;15:431-6.
[12] Nakamoto Y, Mizukoshi E, Tsuji H, Sakai Y, Kitahara M, Arai K, et al. Combined therapy of transcatheter hepatic arterial embolization with intratumoral dendritic cell infusion for hepatocellular carcinoma: clinical safety. Clin Exp Immunol 2007; 147:296-305.

[13] Nakamoto Y, Mizukoshi E, Kitahara M, Arihara F, Sakai Y, Kakinoki K, et al. Prolonged recurrence-free survival following OK432-stimulated dendritic cell transfer into hepatocellular carcinoma during transarterial embolization. Clin Exp Immunol 2011:163:165-77.

[14] Korangy F, Hochst B, Manns MP, Greten TF. Immunotherapy of hepatocellular carcinoma. Expert Rev Gastroenterol Hepatol 2010;4:345-53.

[15] Gottfried E, Kreutz M, Mackensen A. Tumor-induced modulation of dendritic cell function. Cytokine Growth Factor Rev 2008;19:65-77.

[16] Auffermann-Gretzinger S, Keeffe EB, Levy S. Impaired dendritic cell maturation in patients with chronic, but not resolved, hepatitis $C$ virus infection. Blood 2001;97:3171-6.

[17] Ninomiya T, Akbar SM, Masumoto T, Horiike N, Onji M. Dendritic cells with immature phenotype and defective function in the peripheral blood from patients with hepatocellular carcinoma. J Hepatol 1999;31:323-31.

[18] Trinchieri G. Interleukin-12: a cytokine produced by antigen-presenting cells with immunoregulatory functions in the generation of T-helper cells type 1 and cytotoxic lymphocytes. Blood 1994;84:4008-27.

[19] Fujimoto T, Duda RB, Szilvasi A, Chen X, Mai M, O'Donnell MA. Streptococcal preparation OK-432 is a potent inducer of IL-12 and a T helper cell 1 dominant state. J Immunol 1997;158:5619-26.

[20] Katano M, Morisaki T. The past, the present and future of the OK-432 therapy for patients with malignant effusions. Anticancer Res 1998;18:3917-25.

[21] Kuroki H, Morisaki T, Matsumoto K, Onishi H, Baba E, Tanaka M, et al. Streptococcal preparation OK-432: a new maturation factor of monocyte-derived dendritic cells for clinical use. Cancer Immunol Immunother 2003:52:561-8.

[22] Jonuleit H, Kuhn U, Muller G, Steinbrink K, Paragnik L, Schmitt E, et al. Proinflammatory cytokines and prostaglandins induce maturation of potent immunostimulatory dendritic cells under fetal calf serum-free conditions. Eur J Immunol 1997:27:3135-42.

[23] Scandella E, Men Y, Gillessen S, Forster R, Groettrup M. Prostaglandin E2 is a key factor for CCR7 surface expression and migration of monocyte-derived dendritic cells. Blood 2002;100:1354-61. 